\title{
NONHSAT076754 aids ultrasonography in predicting lymph node metastasis and promotes migration and invasion of papillary thyroid cancer cells
}

\author{
Shujun $\mathrm{Xia}^{1}$, Chuandong Wang ${ }^{2}$, Xiaofeng $\mathrm{Ni}^{1}$, Zhongxin $\mathrm{Ni}^{1}$, Yijie Dong ${ }^{1}$ and \\ Weiwei Zhan ${ }^{1}$ \\ ${ }^{1}$ Ultrasound Department, Rui Jin Hospital Shanghai Jiao Tong University School of Medicine, Shanghai, P. R. of China \\ 2 The Key Laboratory of Stem Cell Biology, Institute of Health Sciences, Shanghai Jiao Tong University School of Medicine \& \\ Shanghai Institutes for Biological Sciences, Chinese Academy of Sciences, Shanghai, P. R. of China
}

Correspondence to: Weiwei Zhan, email: shanghairuijinus@163.com

Keywords: NONHSAT076754, papillary thyroid cancer, lymph node metastasis, ultrasonography, biomarker

Received: June 29, 2016

Accepted: November 22, 2016

Published: November 30, 2016

\section{ABSTRACT}

Lymph node metastasis (LNM) is the primary challenge in papillary thyroid cancer (PTC). Recurrent cancerous lymph nodes require repeated surgeries, which increases the risk for surgical complications. Thus, the evaluation of LNM before surgery is important. Ultrasonography is the most convenient way to examine cervical LNM, but the sensitivity of ultrasonography in the identification of LNM in cases of PTC is extremely low. A series of IncRNAs (long noncoding RNAs) have been reported as candidate biomarkers in a variety of tumors. This study detected the IncRNA NONHSAT076754 in PTC and analyzed the correlation of NONHSAT076754 with the clinicopathological and ultrasonographic characteristics of patients with PTC. The value of NONHSAT076754 as an auxiliary diagnostic biomarker for use along with ultrasonography in the differentiation of LNM in PTC was assessed. Additionally, the biological function of NONHSAT076754 in PTC cells was demonstrated. Our study indicated that NONHSAT076754 promotes migration and invasiveness of PTC and serves as a valuable auxiliary biomarker that can be used along with ultrasonography in the prediction of cervical LNM.

\section{INTRODUCTION}

Thyroid cancer is recognized as a common endocrine malignancy. Its incidence has been rapidly increasing in recent years because of more accurate ultrasonographic technology and the application of ultrasound-guided fine-needle aspiration (US-FNA) [1-4]. Papillary thyroid cancer (PTC), which accounts for the majority of cases of thyroid cancer with a good prognosis, is most likely to result in regional lymph node recurrence $[5,6]$. Even though recurrent lymph node metastasis (LNM) is not lethal, it is one of the major challenges in the management of patients with PTC [7]. Repetitive surgeries may increase the risk of surgical complications, and radioiodine ablation may not be effective since some patients do not exhibit sufficient radioiodine uptake or they demonstrate positive metastasis by $18 \mathrm{~F}$-fluorodeoxyglucose positron emission tomography (18FDG-PET) [8, 9]. LNM in PTC is not only an indicator of recurrence but is also used as a reference for the surgical management of patients before surgery [10]. Detection by ultrasound is the most convenient way to examine LNM in PTC, but its sensitivity remains extremely low [11, 12].

Long noncoding RNAs (lncRNAs), which comprise a class of long RNAs (more than 200 nucleotides) without protein coding potential, are involved in various biological processes, including cell proliferation, differentiation, apoptosis, development and immune responses [13, 14]. Some recent reports have demonstrated that lncRNAs play a critical role in cancer development and progression and that they have the potential to serve as diagnostic and prognostic biomarkers in gastric cancer [15], prostate cancer [16], and hepatocellular carcinoma [17], among other cancers. LncRNAs are tumor-specific and are also very stable in serum, urine and other easily obtainable body fluids [18], which indicates that they are good candidate tumor biomarkers. However, conclusions about the diagnostic value of lncRNAs in thyroid cancer are lacking. 
Table 1: Patient demographics

\begin{tabular}{|c|c|c|c|c|}
\hline Clinicopathological characteristics & All patients, $n(\%)$ & $\operatorname{LNM}(n=37)$ & $\operatorname{nonLNM}(n=35)$ & $P$ value \\
\hline Sex & & & & 0.900 \\
\hline Male & $16(22.2 \%)$ & $8(21.6 \%)$ & $8(22.9 \%)$ & \\
\hline Female & $56(77.8 \%)$ & $29(78.4 \%)$ & $27(77.1 \%)$ & \\
\hline $\operatorname{Age}($ mean \pm SD $)$ & & $45.70 \pm 13.25$ & $46.14 \pm 10.66$ & 0.877 \\
\hline$<45 \mathrm{y}$ & $31(43.1 \%)$ & $16(43.2 \%)$ & $15(42.9 \%)$ & \\
\hline$\geq 45 \mathrm{y}$ & $41(56.9 \%)$ & $21(56.8 \%)$ & $20(57.1 \%)$ & \\
\hline Extrathyroid extension & & & & 0.327 \\
\hline Presence & $1(1.4 \%)$ & $1(2.7 \%)$ & $0(0.0 \%)$ & \\
\hline Absence & $71(98.6 \%)$ & $36(97.3 \%)$ & $35(100.0 \%)$ & \\
\hline Multifocality & & & & 0.071 \\
\hline Unifocal & $53(73.6 \%)$ & $24(64.9 \%)$ & $29(82.9 \%)$ & \\
\hline Multifocal & $19(26.4 \%)$ & $13(35.1 \%)$ & $6(17.1 \%)$ & \\
\hline Tumor(T) & & & & 0.974 \\
\hline T1a & $35(48.6 \%)$ & $15(40.5 \%)$ & $20(57.1 \%)$ & \\
\hline $\mathrm{T} 1 \mathrm{~b}$ & $37(56.4 \%)$ & $22(59.5 \%)$ & $15(42.9 \%)$ & \\
\hline Lymph node(N) & & & & 1 \\
\hline N0 & $35(48.6 \%)$ & 1 & 1 & \\
\hline N1a & $24(33.3 \%)$ & 1 & 1 & \\
\hline N1b & $13(18.1 \%)$ & 1 & 1 & \\
\hline \multicolumn{5}{|l|}{ Metastasis(M) } \\
\hline M0 & $72(100 \%)$ & 1 & 1 & 1 \\
\hline \multicolumn{5}{|l|}{ pTNM stage } \\
\hline Early(I/II) & $50(69.4 \%)$ & $15(40.5 \%)$ & $35(100.0 \%)$ & $0.000 *$ \\
\hline Advanced(III/IV) & $22(30.6 \%)$ & $22(59.5 \%)$ & $0(0.0 \%)$ & \\
\hline
\end{tabular}

$* P<0.05 ; \mathrm{LNM}=$ lymph node metastasis

The lncRNA NONHSAT076754 is a transcript of fibronectin 1, which is a cell surface and extracellular protein that is involved in cell adhesion and migration processes including those of wound healing [19], blood coagulation [20], and metastasis [21], among others. The present study investigated the expression of NONHSAT076754 in PTC and its correlation with patient characteristics. The diagnostic value of NONHSAT076754 and ultrasonography in the identification of LNM in PTC was analyzed in clinical samples. Moreover, the biological function of NONHSAT076754 was assessed in PTC cell lines. This study aims to identify NONHSAT076754 as an auxiliary biomarker for use with ultrasonography in the prediction of LNM and its role in PTC.

\section{RESULTS}

\section{Patient demographics}

As shown in Table 1, 72 patients were included in our study. They were classified into two groups (LNM group, $n=37$; nonLNM group, $n=35$ ) according to whether the patients had LNM. The distribution of sex, age and multifocality in the two groups was the same $(\mathrm{P}>0.05)$.
Among all these patients, only one (1.4\%) patient who was in the LNM group exhibited extrathyroid extension. According to the American Joint Committee on Cancer (AJCC) TNM staging system ( $7^{\text {th }}$ edition), the tumors were all designated pT1, and no patients had distant metastasis. In the LNM group, $64.9 \%$ of the patients were N1a and $35.1 \%$ were N1b. Patients with LNM were more likely to be in an advanced stage of the disease (III/IV) $(P<0.05)$.

\section{NONHSAT076754 overexpression is associated with LNM in PTC}

The expression level of NONHSAT076754 was detected in tumor tissues of the 72 patients. NONHSAT076754 was significantly up-regulated in 37 metastatic PTCs compared with 35 nonmetastatic PTCs (Figure 1A). We further analyzed the correlation of NONHSAT076754 expression and the clinicopathological and ultrasonographic characteristics of all these patients. Furthermore, the expression level of NONHSAT076754 was determined by a receiver operating characteristic (ROC) curve. The cutoff value of NONHSAT076754 expression was calculated by ROC curve analysis. The highest diagnostic value was at a cutoff of $\Delta C t=4.152$, according to which all PTC patients were categorized into 
Table 2: Correlations between NONHSAT076754 expression and clinicopathological characteristics of patients with PTC

\begin{tabular}{|c|c|c|c|c|}
\hline Clinicopathological characteristics & $n$ & High expression & Low expression & $P$ value \\
\hline Sex & & & & 0.576 \\
\hline Male & 16 & $7(18.9 \%)$ & $9(25.7 \%)$ & \\
\hline Female & 56 & $30(81.1)$ & $26(74.3 \%)$ & \\
\hline \multicolumn{5}{|l|}{ Age } \\
\hline$<45 y$ & 31 & $14(37.8 \%)$ & $17(48.6 \%)$ & 0.476 \\
\hline$\geq 45 y$ & 41 & $23(62.2 \%)$ & $18(51.4 \%)$ & \\
\hline Extrathyroid extension & & & & 1.000 \\
\hline Presence & 1 & $1(2.7 \%)$ & $0(0.0 \%)$ & \\
\hline Absence & 71 & $36(97.3 \%)$ & $35(100 \%)$ & \\
\hline Multifocality & & & & 1.000 \\
\hline Unifocal & 53 & $27(73.0 \%)$ & $26(74.3 \%)$ & \\
\hline Multifocal & 19 & $10(27.0 \%)$ & $9(25.7 \%)$ & \\
\hline Tumor(T) & & & & 0.098 \\
\hline T1a & 35 & $14(37.8 \%)$ & $21(60.0 \%)$ & \\
\hline $\mathrm{T} 1 \mathrm{~b}$ & 37 & $23(62.2 \%)$ & $14(40.0 \%)$ & \\
\hline Lymph node(N) & & & & $0.009 *$ \\
\hline N0 & 35 & $12(32.4 \%)$ & $23(65.7 \%)$ & \\
\hline N1a & 24 & $18(48.6 \%)$ & $6(17.1 \%)$ & \\
\hline N1b & 13 & $7(18.9 \%)$ & $6(17.1 \%)$ & \\
\hline Metastasis(M) & & & & 1 \\
\hline M0 & $72(100 \%)$ & 1 & 1 & \\
\hline pTNM stage & & & & $0.022 *$ \\
\hline Early(I/II) & 50 & $21(56.8 \%)$ & $29(82.9 \%)$ & \\
\hline Advanced(III/IV) & 22 & $16(43.2 \%)$ & $6(17.1 \%)$ & \\
\hline
\end{tabular}

$* P<0.05$

either the low-expression group $(n=35, \Delta C t>4.152)$ or the high-expression group $(n=37, \Delta C t<4.152)$. As summarized in Table 2, NONHSAT076754 overexpression was significantly correlated with LNM in PTC (N0 vs N1a\&N1b, $P=0.009$ ) as well as with pTNM stage (I-II vs III-IV, $P=0.022$ ). However, no significant associations were observed between NONHSAT076754 expression and sex $(P=0.576)$, age $(P=0.476)$, extrathyroid extension $(P=1.000)$, multifocality $(P=1.000)$ or tumor stage $(P=0.098)$. For the ultrasonographic characteristics, no significant differences were observed between the two groups (Table 3). Moreover, the univariate and multivariate logistic regression analyses showed that NONHSAT076754 overexpression was a strong independent diagnostic factor for LNM in patients with PTC $(P=0.000)$ (Table 4).

\section{Combined diagnostic value of NONHSAT076754 with ultrasonography of LNM in PTC}

To further evaluate the possibility of the clinical application of NONHSAT076754 in patients with PTC, the diagnostic potential and discriminatory accuracy of ultrasonography-NONHSAT076754 was evaluated by
ROC curve analysis and the corresponding area under the curve (AUC) values. The AUC for NONHSAT076754 was $0.878(95 \%$ confidence interval $(\mathrm{CI})=0.798-0.958$; $P<0.001$, Figure 1D). The level of NONHSAT076754 overexpression showed a sensitivity of $83.78 \%$ and a specificity of $82.85 \%$ with a diagnostic accuracy of $83.33 \%$, whereas the sensitivity and specificity of ultrasonography were $35.14 \%$ and $88.57 \%$, respectively, with a diagnostic accuracy of $61.11 \%$. The combined diagnostic value of ultrasonography-NONHSAT076754 was increased; the sensitivity was $91.89 \%$, the specificity was $82.85 \%$, and the accuracy was $87.50 \%$. (Table 5)

\section{The expression and subcellular distribution of NONHSAT076754 in PTC cell lines}

The expression of NONHSAT076754 was further confirmed in two PTC cell lines (TPC1, K1) by reverse transcription-quantitative polymerase chain reaction (RT-qPCR). The results showed that the expression of NONHSAT076754 was significantly higher in the K1 cell line than in the TPC1 cell line (Figure 1B). The results obtained in cell lines were consistent with those obtained in patient samples. Additionally, the fluorescence in situ 
Table 3: Correlations between NONHSAT076754 expression and ultrasonographic characteristics of PTC

\begin{tabular}{|c|c|c|c|c|}
\hline Ultrasonographic characteristics & $n$ & High expression & Low expression & $P$ value \\
\hline Border & & & & 0.788 \\
\hline Clear & 18 & $10(27.0 \%)$ & $8(22.9 \%)$ & \\
\hline Obscure & 54 & $27(73.0 \%)$ & $27(77.1 \%)$ & \\
\hline Margin & & & & 0.795 \\
\hline Regular & 20 & $11(29.7 \%)$ & $9(25.7 \%)$ & \\
\hline Irregular & 52 & $26(70.3 \%)$ & $26(74.3 \%)$ & \\
\hline Shape & & & & 1.000 \\
\hline Taller than wide & 6 & $3(8.1 \%)$ & $3(8.6 \%)$ & \\
\hline Wider than tall & 66 & $34(91.9 \%)$ & $32(91.4 \%)$ & \\
\hline Halo & & & & 0.540 \\
\hline Present & 7 & $4(10.8 \%)$ & $3(8.6 \%)$ & \\
\hline Absent & 65 & $33(89.2 \%)$ & $32(91.4 \%)$ & \\
\hline Internal architecture & & & & 0.240 \\
\hline Solid & 69 & $34(91.9 \%)$ & $35(100.0 \%)$ & \\
\hline Mostly solid & 3 & $3(8.1 \%)$ & $0(0.0 \%)$ & \\
\hline Echogenicity & & & & 0.984 \\
\hline Markedly hypoechoic & 18 & $9(24.3 \%)$ & $9(25.7 \%)$ & \\
\hline Hypoechoic & 46 & $24(64.9 \%)$ & $22(62.9 \%)$ & \\
\hline Isoechoic & 8 & $4(10.8 \%)$ & $4(11.4 \%)$ & \\
\hline Echo homogeneity & & & & 0.460 \\
\hline Homogenous & 24 & $14(37.8 \%)$ & $10(28.6 \%)$ & \\
\hline Inhomogeneous & 48 & $23(62.2 \%)$ & $25(71.4 \%)$ & \\
\hline Calcification & & & & 0.329 \\
\hline None & 31 & $14(37.8 \%)$ & $17(48.6 \%)$ & \\
\hline Micro & 33 & $20(54.1 \%)$ & $13(37.1 \%)$ & \\
\hline Macro & 8 & $3(8.1 \%)$ & $5(14.3 \%)$ & \\
\hline Contact & & & & 0.123 \\
\hline $0-25 \%$ & 57 & $27(73.0 \%)$ & $30(85.7 \%)$ & \\
\hline $25-50 \%$ & 11 & $6(16.2 \%)$ & $5(14.3 \%)$ & \\
\hline$>50 \%$ & 4 & $4(10.8 \%)$ & 0 & \\
\hline Lateral shadow & & & & 0.486 \\
\hline Absence & 71 & $37(100.0 \%)$ & $34(97.1 \%)$ & \\
\hline Presence & 1 & 0 & $1(2.9 \%)$ & \\
\hline Blood flow & & & & 0.411 \\
\hline None & 25 & $10(27.0 \%)$ & $15(42.9 \%)$ & \\
\hline Low & 23 & $12(32.4 \%)$ & $11(31.4 \%)$ & \\
\hline Medium & 14 & $8(21.6 \%)$ & $6(17.1 \%)$ & \\
\hline High & 10 & $7(18.9 \%)$ & $3(8.6 \%)$ & \\
\hline Vascularity & & & & 0.412 \\
\hline None & 24 & $11(29.7 \%)$ & $13(37.1 \%)$ & \\
\hline Circular & 19 & $8(21.6 \%)$ & $11(31.4 \%)$ & \\
\hline Central & 1 & $1(2.7 \%)$ & $0(0.0 \%)$ & \\
\hline Mix & 28 & $17(45.9 \%)$ & $11(31.4 \%)$ & \\
\hline
\end{tabular}


Table 4: Univariate and multivariate regression analyses of parameters associated with LNM

\begin{tabular}{|c|c|c|c|c|c|}
\hline \multirow[t]{2}{*}{ Parameters } & \multirow[t]{2}{*}{ Category } & \multicolumn{2}{|c|}{ Univariate analysis } & \multicolumn{2}{|c|}{ Multivariate analysis } \\
\hline & & OR(95\% CI) & $P$ value & OR(95\% CI) & $P$ value \\
\hline Sex & Male/Female & $\begin{array}{l}0.484 \\
(0.155-1.515)\end{array}$ & 0.212 & & \\
\hline Age & $<45 / \geq 45 y$ & $\begin{array}{l}1.016 \\
(0.399-2.583)\end{array}$ & 0.974 & & \\
\hline Multifocality & Unifocal/Multifocal & \begin{tabular}{|l|}
0.700 \\
$(0.243-2.017)$
\end{tabular} & 0.509 & & \\
\hline Tumor size & $\mathrm{T} 1 \mathrm{a} / \mathrm{T} 1 \mathrm{~b}$ & \begin{tabular}{|l}
0.511 \\
$(0.200-1.306)$ \\
\end{tabular} & 0.161 & & \\
\hline Stage & Early/Advanced & $\begin{array}{l}1.136 \\
(1.04-1.464)\end{array}$ & 0.001 & $\begin{array}{l}1.149 \\
(1.031-1.703)\end{array}$ & $0.016^{*}$ \\
\hline NONHSAT076754 & Low/High & $\begin{array}{l}1.040 \\
(1.012-1.138)\end{array}$ & 0.000 & $\begin{array}{l}1.042 \\
(1.011-1.160)\end{array}$ & $0.000 *$ \\
\hline
\end{tabular}

$* P<0.05 ; \mathrm{LNM}=$ lymph node metastasis; $\mathrm{OR}=$ odds ratio; $\mathrm{CI}=$ confidence interval

hybridization (FISH) analysis demonstrated that both TPC1 and K1 cells exhibited positivity in the cytoplasm when a fluorescence-conjugated NONHSAT076754 probe was used, which shows that NONHSAT076754 is a lncRNA that is distributed in the cytoplasm. The fluorescent signal in $\mathrm{K} 1$ cells was much higher than that in TPC1 cells, which also indicates that the expression level of NONHSAT076754 in K1 cells was higher than that in TPC1 cells. (Figure 1C)
A

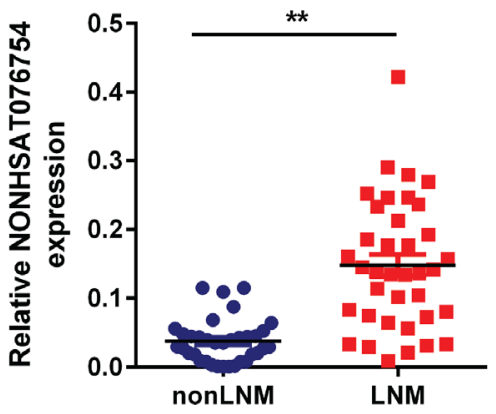

C
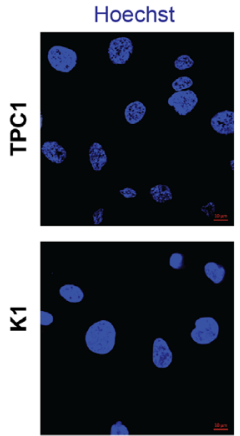

NONHSAT076754
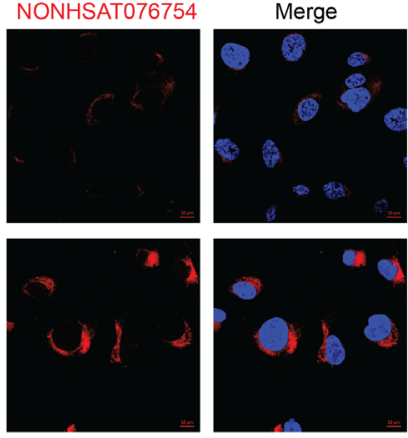

B

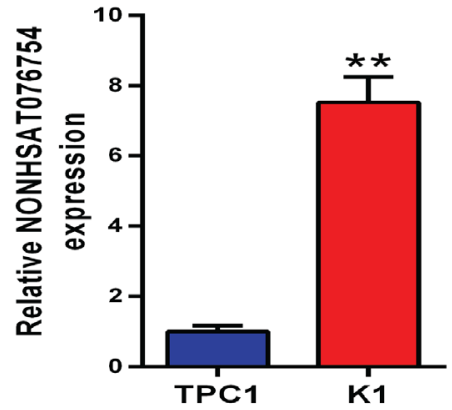

$\mathrm{D}$

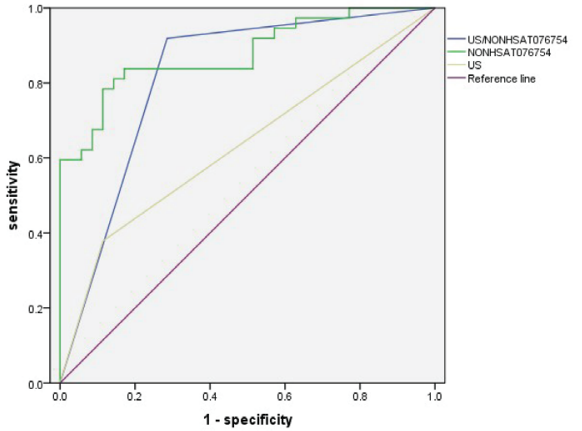

Figure 1: LncRNA NONHSAT076754 is overexpressed in PTC with LNM. The expression level of NONHSAT076754 was detected in PTC tissues (nonLNM $(n=35)$ and LNM $(n=37))$ A. and cell lines B. by qRT-PCR. The value was normalized to GAPDH mRNA expression. "**” indicates $P<0.05$. C. FISH analysis of the subcellular distribution of NONHSAT076754 in TPC1 and K1 cells as detected by confocal microscopy. Nuclei (blue) were labeled with Hoechst solution; the NONHSAT076754 probe (red) was labeled with Cy3. Representative images of three repeated experiments are shown. The scale bar is $10 \mu \mathrm{m}$. D. The ROC curves of ultrasonography and NONHSAT076754 predicted potential diagnostic values of ultrasonography and NONHSAT076754. NONHSAT076754: AUC 0.878 (0.798, 0.958), $\mathrm{P}<0.01$; US/NONHSAT076754: AUC 0.817 (0.712, 0.921), $\mathrm{P}<0.01$. 
Table 5: Performance of US-NONHSAT076754 in the differentiation of LNM from nonLNM in PTC

\begin{tabular}{|l|c|c|c|c|c|c|}
\hline & LNM & Sensitivity & Specificity & NPV & PPV & Accuracy \\
\hline US & +17 & $35.14 \%$ & $88.57 \%$ & $56.36 \%$ & $76.47 \%$ & $61.11 \%$ \\
\hline & -55 & $(13 / 37)$ & $(31 / 35)$ & $(31 / 55)$ & $(13 / 17)$ & $(44 / 72)$ \\
\hline NONHSAT076754 & +37 & $83.78 \%$ & $82.85 \%$ & $82.85 \%$ & $83.78 \%$ & $83.33 \%$ \\
\hline & -35 & $(31 / 37)$ & $(29 / 35)$ & $(29 / 35)$ & $(31 / 37)$ & $(60 / 72)$ \\
\hline \multirow{2}{*}{$\begin{array}{l}\text { US- } \\
\text { NONHSAT076754 }\end{array}$} & +40 & $91.89 \%$ & $82.85 \%$ & $90.62 \%$ & $85.00 \%$ & $87.50 \%$ \\
\cline { 2 - 7 } & -32 & $(34 / 37)$ & $(29 / 35)$ & $(29 / 32)$ & $(34 / 40)$ & $(63 / 72)$ \\
\hline
\end{tabular}

$\mathrm{US}=$ ultrasonography; LNM = lymph node metastasis; NPV = negative predictive value; $\mathrm{PPV}=$ positive predictive value

\section{Overexpression of NONHSAT076754 promotes the migration and invasiveness of TPC1 cells}

A scratch assay was performed to examine the migration ability of TPC1 cells after NONHSAT076754 was overexpressed (OE-NONHSAT076754 group). We prepared stable NONHSAT076754-overexpressing transfectants using a lentiviral system (see supplementary Figure S1A). After transfection, TPC1 cells were plated in a 6 -well plate. When the cells reached $100 \%$ confluence, $1-\mathrm{mL}$ sterile pipet tips were used to scratch the cells.

A

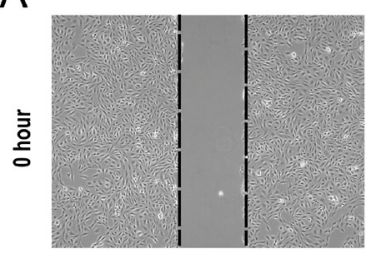

OE-NONHSATO76754

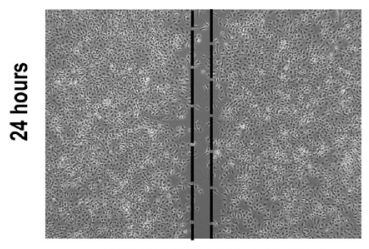

OE-NONHSAT076754

B

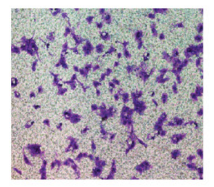

OE-NONHSAT076754

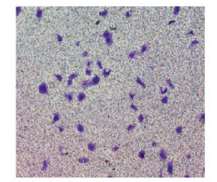

OE-NC

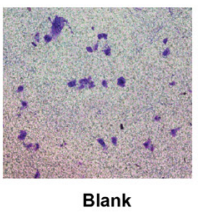

Blank

$\mathrm{D}$

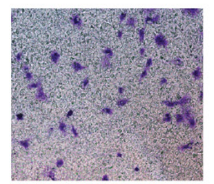

OE-NONHSAT076754

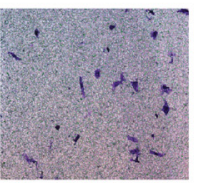

OE-NC
OE-NC

OE-NC

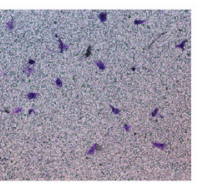

Blank
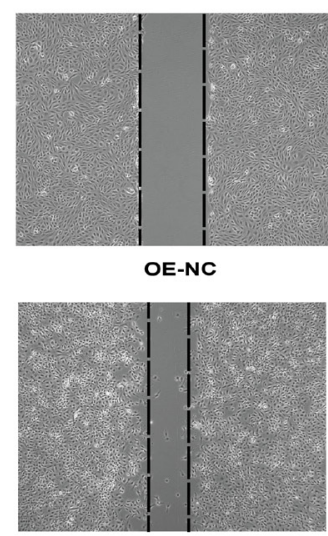

C

E
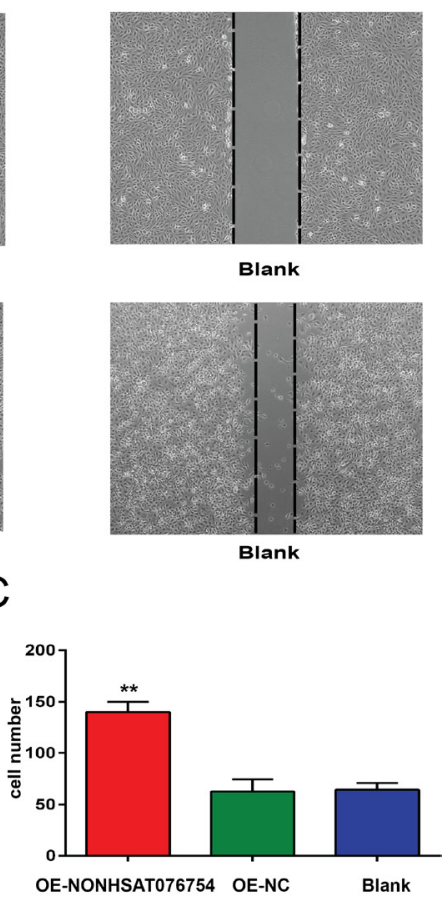

Blank

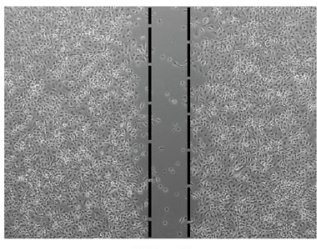

Blank

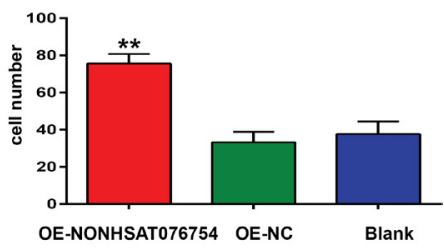

Figure 2: Effect of NONHSAT076754 on the migration and invasiveness of TPC1 cells. A. A scratch test was performed to determine the migration ability of TPC1 cells. Representative images at 0 and 24 hours from three repeated experiments are shown. B. A Transwell assay was performed to determine the migration ability of TPC1 cells. Representative images show invasive cells in the lower chamber stained with crystal violet. $\mathbf{C}$. The quantification of cell migration is presented as the number of migrating cells. Data are expressed as the means \pm SD of three independent experiments. D. A Transwell assay was performed to determine the invasion ability of TPC1 cells. Representative images show invasive cells in the lower chamber stained with crystal violet. $\mathbf{E}$. The quantification of cell invasion is presented as number of migrating cells. Data are expressed as the means $\pm \mathrm{SD}$ of three independent experiments. "***" indicates $P<0.01$. 
After a $24 \mathrm{~h}$ incubation, the distance of the scratch wound in the OE-NONHSAT076754 group was found to be significantly smaller compared with that in the control group (Figure 2A). To further detect the effect of NONHSAT076754 on migration and invasion, a transwell assay was performed in TPC1 cells. The number of cells in the OE-NONHSAT076754 group that migrated through the chamber $(140 \pm 10)$ was significantly higher than the number of cells in the OE-negative control (NC) group $(62.67 \pm 11.68)$ and the Blank group $(64.33 \pm 6.66)$ (Figure $2 \mathrm{~B}, 2 \mathrm{C})$. A similar result was also shown in an invasion assay (Figure 2D, 2E): OE-NONHSAT076754 group: $75.67 \pm 5.13$, OE-NC group: $33.33 \pm 5.51$, Blank group: $37.67 \pm 6.81$. The data indicate that NONHSAT076754 promotes the migration and invasiveness of PTC cells.

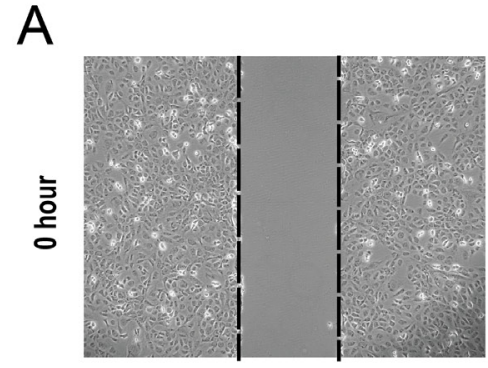

KD-NONHSAT076754

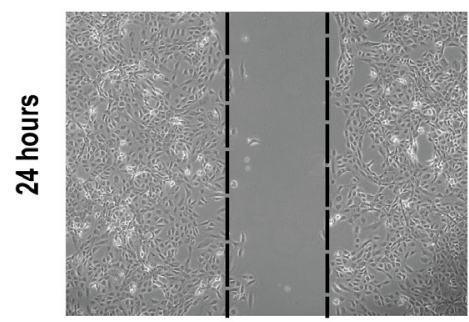

KD-NONHSAT076754

B

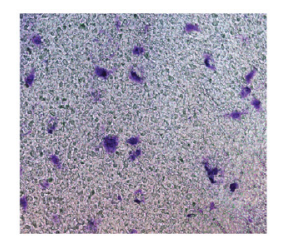

KD-NONHSAT076754

D

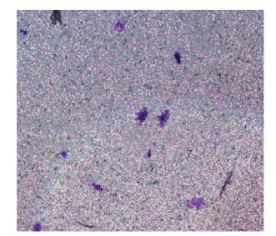

KD-NONHSAT076754

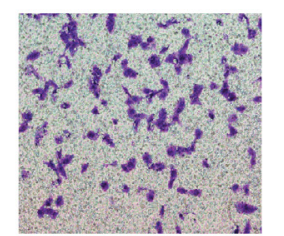

KD-NC

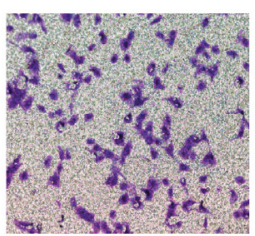

Blank

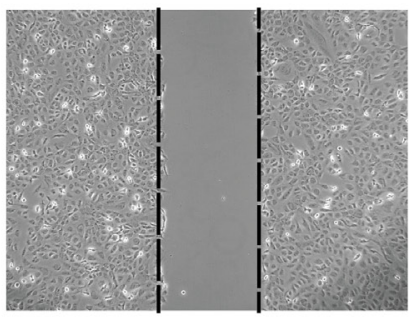

Blank

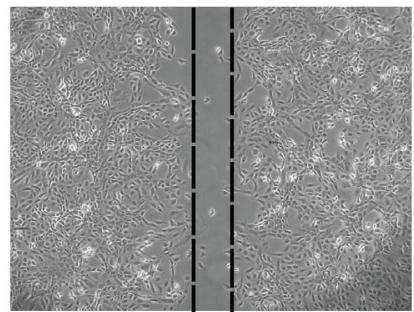

Blank

C

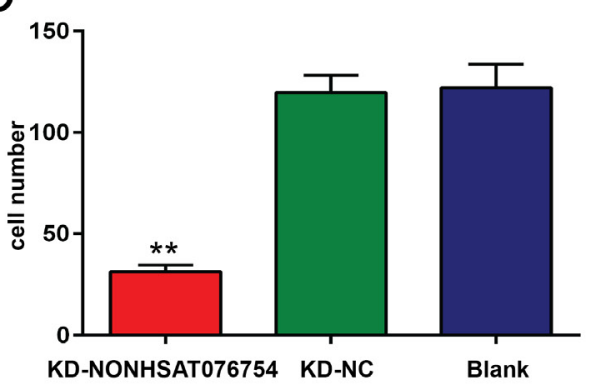

E

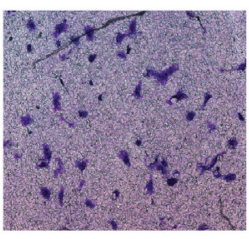

Blank

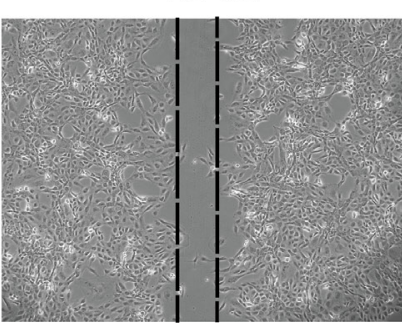

KD-NC

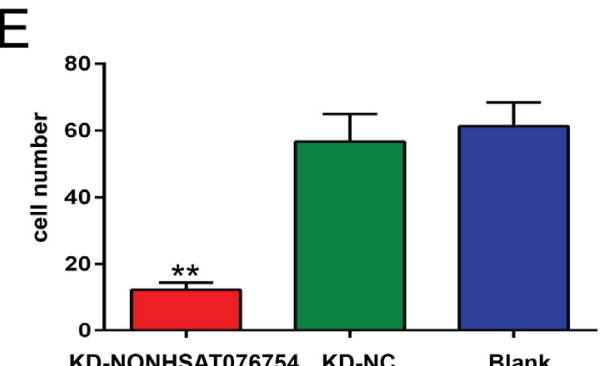

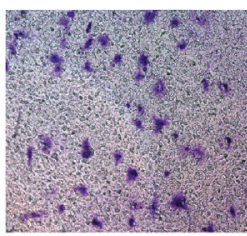

KD-NC

Figure 3: Effect of NONHSAT076754 on the migration and invasiveness of K1 cells. A. A scratch test was performed to determine the migration ability of $\mathrm{K} 1$ cells. Representative images at 0 and 24 hours from three repeated experiments are shown. B. A Transwell assay was performed to determine the migration ability of K1 cells. Representative images show invasive cells in the lower chamber stained with crystal violet. C. The quantification of cell migration is presented as the number of migrating cells. Data are expressed as the means \pm SD of three independent experiments. D. A Transwell assay was performed to determine the invasion ability of K1 cells. Representative images show invasive cells in the lower chamber stained with crystal violet. E. The quantification of cell invasion is presented as the number of migrating cells. Data are expressed as the means \pm SD of three independent experiments. "**" indicates $P<0.01$. 


\section{Knockdown of NONHSAT076754 inhibits the migration and invasiveness of $\mathrm{K} 1$ cells}

Transient knockdown of NONHSAT076754 expression was achieved using antisense oligonucleotides (see supplementary Figure S1B). Scratch and Transwell assays were also performed. $\mathrm{K} 1$ cells were plated in a 6-well plate and were cells were scratched by a $1-\mathrm{mL}$ sterile pipet tip when they reached $100 \%$ confluence. After a 24-h incubation, the distance of the scratch wound in the KD-NONHSAT076754 group was significantly larger than that in the control group (Figure 3A). Moreover, a Transwell assay was also performed in K1 cells. The number of KD-NONHSAT076754 cells that migrated through the chamber $(31.33 \pm 3.21)$ was significantly lower than the number of KD-NC cells $(119.67 \pm 8.50)$ and cells in the Blank group (122 \pm 11.53 ) (Figure 3B, 3C). A similar result was also obtained in the invasion assay (Figure 3D, 3E): KD-NONHSAT076754 group: $12.33 \pm 2.08$, KD-NC group: $50.67 \pm 8.33$, Blank group: $61.33 \pm 7.09$. The data indicate that NONHSAT076754 inhibits the migration and invasiveness of PTC cells.
A

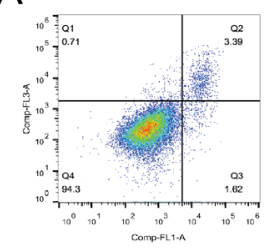

OE-NONHSAT076754

C

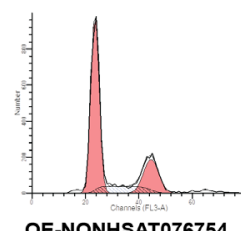

$E$

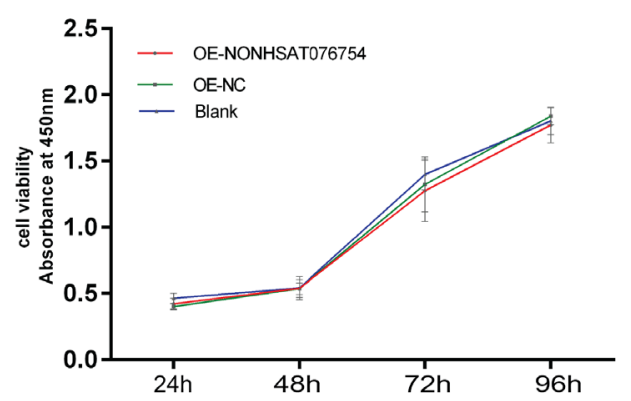

G

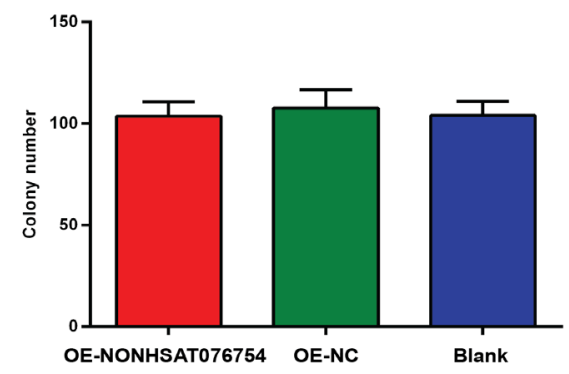

B

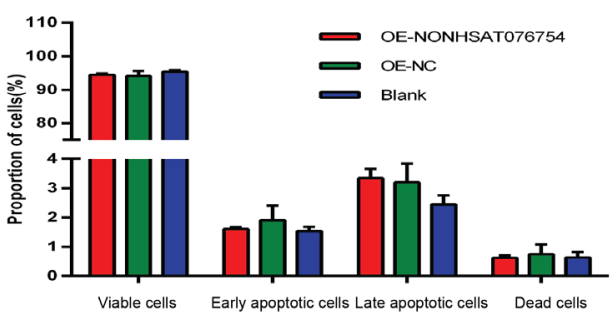

D

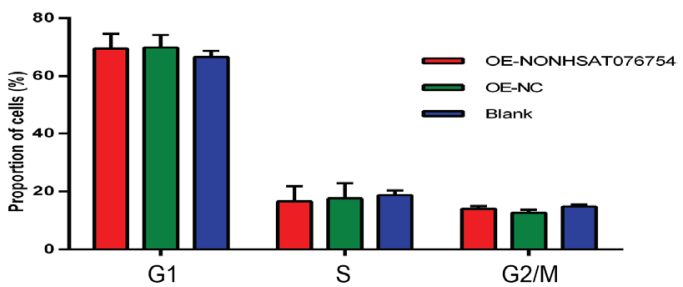

$\mathrm{F}$

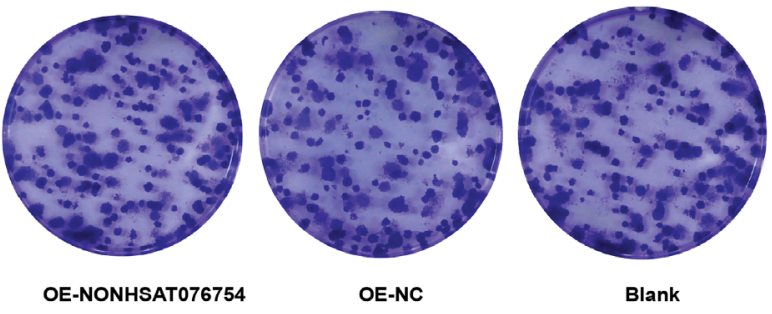

OE-NONHSAT076754 OE-NC Blank

Figure 4: Effect of NONHSAT076754 on the proliferation and cell cycle status of TPC1 cells. A. Images from the flow cytometric analysis of apoptosis in TPC1 cells. B. The percentage of apoptotic cells is presented in the histogram. Data are expressed as the means \pm SD of three independent experiments. C. Images from the flow cytometric analysis of the cell cycle in TPC1 cells. D. Results quantified in the cell cycle analysis are shown as a percentage of the total number of cells. Data are expressed as the means \pm SD of three independent experiments. E. A CCK-8 assay was performed to determine the proliferation of TPC1 cells transfected with NONHSAT076754 after 24 hours, 48 hours, 72 hours and 96 hours. F. A colony formation assay was performed to determine the proliferation of TPC1 cells transfected with NONHSAT076754. The colonies were captured and counted. G. The colony formation assay results are presented in the histogram. All experiments were performed independently in triplicate, and the results are presented as the means \pm SD. 


\section{Effect of NONHSAT076754 on apoptosis, the cell cycle and cell proliferation in PTC}

Flow cytometry, which was used to detect the level of apoptosis, and cell cycle analysis were performed to detect the influence of NONHSAT076754 on the apoptosis and the cell cycle status of PTC cells. According to the flow cytometric apoptosis analysis, signals from apoptotic cells and viable cells were similar in the OE-NONHSAT076754 group and the control groups (Figure 4A, 4B). This result was consistent between the KD-NONHSAT076754 group and the control groups (see supplementary Figure S2A, S2B). Cell cycle analysis showed that the proportion of cells in the OE-NONHSAT076754 group that were in $\mathrm{G} 1, \mathrm{~S}$, and $\mathrm{G} 2 / \mathrm{M}$ phases were similar to those in the OE-NC and Blank groups (Figure 4C, 4D). Similarly, no difference was observed in the proportion of cells in the KD-NONHSAT076754 group, the KD-NC group and the Blank group in each phase (see supplementary Figure S2C, S2D).

In the CCK8 assay, the proliferation abilities of $\mathrm{TPC} 1$ and $\mathrm{K} 1$ cells were examined at four time points (24 h, $48 \mathrm{~h}, 72 \mathrm{~h}, 96 \mathrm{~h}$ ) after transfection. The cell viabilities are shown in a proliferation curve, which was generated based on the absorbance at $450 \mathrm{~nm}$. The proliferation ability of cells in the OE-NONHSAT076754 group was similar to that in the control groups (Figure 4E). The same variation trend was found in K1 cells (see supplementary Figure S2E). In addition, the impact of NONHSAT076754 on the proliferation of TPC1 and $\mathrm{K} 1$ cells was determined by colony formation assay. In the colony formation assay, the number of clones was not different between the OENONHSAT076754 group and the control groups (Figure 4F, 4G) and a similar variation was found in K1 cells (see supplementary Figure S2F, S2G). The results indicate that apoptosis, the cell cycle and proliferation ability are not affected by NONHSAT076754.

\section{DISCUSSION}

In this study, NONHSAT076754 was significantly increased in PTC patients with LNM compared with PTC patients without LNM. Our study indicated for the first time that increased NONHSAT076754 expression is associated with LNM of PTC and advanced pTNM stage. Our study also analyzed the diagnostic value of ultrasonography and NONHSAT076754 in the differentiation of LNM in PTC. The sensitivity of ultrasonography was very low, while the specificity was high, which was consistent with previous reports [22]. The sensitivity was highly increased when NONHSAT076754 and ultrasonography were combined for diagnosis, which allowed the diagnostic value to increase substantially. Moreover, the in vitro function study demonstrated that NONHSAT076754 promotes the migration and invasiveness of PTC cells. This study, for the first time, identified NONHSAT076754 as a valuable clinical biomarker due to its ability to complement ultrasonography in the prediction of LNM in PTC.

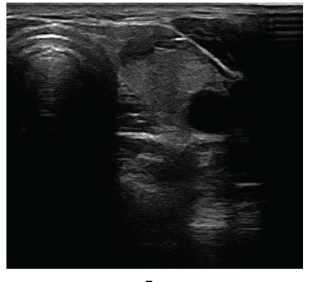

A

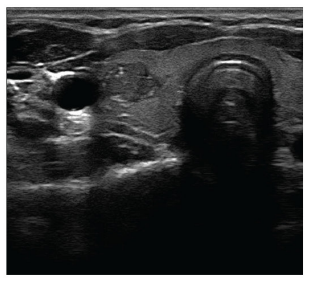

C

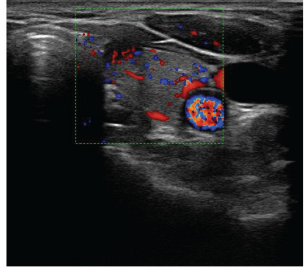

B

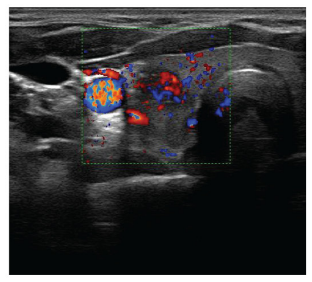

D

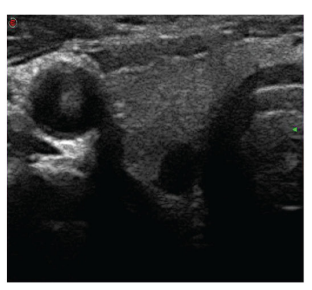

E

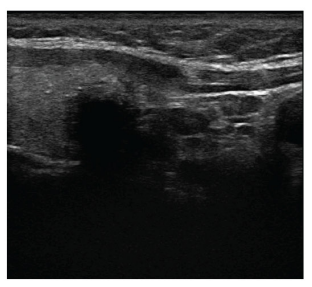

G

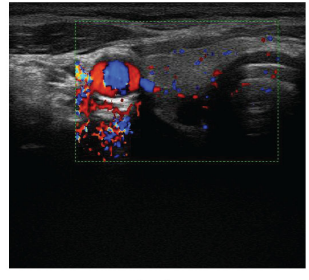

$\mathrm{F}$

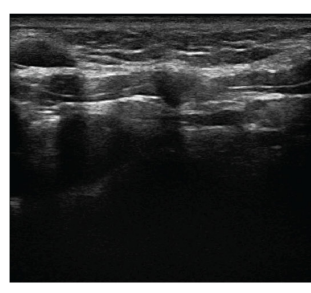

$\mathrm{H}$

Figure 5: A.\&B. A hypoechoic tumor on the left lobe with an irregular margin and 25 50\% capsular abutment. Color-Doppler showed central vascularity with medium blood flow. LNM in the left central compartment was detected by ultrasonography while pathology showed no cervical LNM. C.\&D. An isoechoic tumor on the right lobe with an irregular margin and microcalcification. Color-Doppler showed mixed vascularity with high blood flow. LNM in both left and right central compartment was detected by ultrasonography while LNM only on the right side was confirmed by pathology. E.\&F. A markedly hypoechoic tumor on the right lobe with an irregular margin and low blood flow. No suspected LNM was detected by ultrasonography while LNM in the right central compartment were diagnosed by pathology. G.\&H. A hypoechoic tumor on the right lobe with an irregular margin. LNM in the right central compartment was detected by ultrasonography while pathology confirmed LNM in both the left and right central compartments. 
LNM in PTC is not fatal, but the presence of LNM would definitely determine whether the surgeon should perform lymph node dissection or recommend $\mathrm{I}^{131}$ therapy and assists in the prediction of the recurrence and prognosis of patients with PTC. Thus, it is important to obtain information on lymph node status before surgery is performed. Ultrasound imaging, as the primary method used for the preoperative assessment of cervical lymph nodes, has a low sensitivity. The way in which cervical lymph node status is evaluated needs to be optimized. Tumor biomarkers are a useful tool in the diagnosis and postoperative surveillance in various cancers [23-27]. However, biomarkers of LNM in PTC have never been reported. This is the first study that presents a LNMassociated biomarker for PTC.

The emerging field of lncRNAs has indicated their clinical potential with respect to a better understanding and management of different types of tumors. It was reported in previous studies that specific lncRNAs might be potent prognostic and predictive biomarkers [28, 29]. LncRNAs do have some advantages as clinical biomarkers. First, lncRNAs are considered more tissue-specific than proteincoding genes. It was found that the lncRNA PCA3 in urine has a higher specificity and sensitivity than serum PSA in prostate cancer patients $[16,30]$. Second, circulating RNA, which is mostly derived from tumor secretions, remains quite stable under extreme conditions in the blood, despite low $\mathrm{pH}$ and the presence of enzymes, because of the protection afforded by membranous vesicles [18, 31]. LncRNA is an applicable biomarker in blood samples, which are easy to obtain before surgery. Furthermore, serum IncRNA is able to be tested by traditional PCR, which is the most convenient and simple laboratory technology available [17].

This study investigated the clinical relevance of NONHSAT076754 in PTC. Our study confirmed the correlation of NONHSAT076754 with LNM in PTC. NONHSAT076754 is positively associated with LNM in PTC. When combined with the detection of NONHSAT076754, the diagnostic value of ultrasonography was also greatly increased for the differentiation of PTC with LNM from PTC without LNM. Ultrasonography is a useful tool for the identification and location of metastasis in the neck; however, for lymph nodes without typical signs of metastasis (e.g., microcalcification, cystic changes) [32], it is difficult to make a decision regarding surgery through ultrasonography alone. NONHSAT076754 is of great value for the indication of the existence of LNM in PTC. Using ultrasonography and NONHSAT076754 together would reduce the rate of missed diagnoses.

Recently, accumulating evidence suggests that lncRNAs are deeply involved in cellular activities such as cell growth, proliferation, and apoptosis as well as migration and invasion through the regulation of specific genes, RNAs or proteins. Lnc-CC3 was reported to increase cervical cancer metastasis through the upregulation of Slug expression [33]. The lncRNA GClnc1, which has been functionally and clinically demonstrated to be a novel oncogene, promotes gastric carcinogenesis and may act as a modular scaffold for WDR5 and KAT2A complexes to specify the histone modification pattern [34]. It was also reported that the upregulation of the long non-coding RNA AGAP2-AS1 facilitates cell proliferation, migration and invasion, and inhibits cell apoptosis; it also represses LATS2 and KLF2 expression through its interaction with EZH2 and LSD1 in non-small-cell lung cancer cells [35]. Our study illustrated the critical function of the lncRNA NONHSAT076754 in PTC cell migration and invasion, even though this lncRNA does not influence the viability, cell cycle status or apoptosis of PTC cells. Our result indicated that NONHSAT076754 plays a critical role in PTC metastasis and that it promotes PTC progression, although the mechanism of how NONHSAT076754 regulates PTC metastasis has not been elucidated.

In conclusion, NONHSAT076754, which is associated with LNM in PTC, promotes migration and invasiveness in PTC. The addition of NONHSAT076754 led to an increase in the diagnostic value of ultrasonography. Therefore, NONHSAT076754 is a valuable auxiliary diagnostic biomarker that can be used along with ultrasonography for the prediction of cervical LNM in PTC.

\section{SUBJECTS AND METHODS}

\section{Ultrasound examination}

Ultrasound examination of the thyroid was performed with a 5-12 MHz linear array probe. Philips iU22 (Philips Ultrasound, USA) and ESAOTE My Lab 90 (ESAOTE, Italy) ultrasound diagnostic equipment were used for the analysis. Patients were in a supine position, and the anterior neck area was fully exposed. Transverse and longitudinal scanning of each nodule was performed by two radiologists with more than 10 years of experience in thyroid ultrasound. The ultrasonographic features of the thyroid tumors were observed in greyscale. The border (clear/obscure), margin (regular/irregular), shape, peripheral halo, internal architecture (solid/mostly solid), calcification (none/micro/macro), echogenicity, echo homogeneity, lateral shadow (presence/absence), and the degree of capsular contact were recorded. Color-Doppler ultrasound was also included to analyze the blood flow (none/ low/medium/high) and vascularity (none/circular/ central/mix) of the nodules. In patients with multifocal tumors, the most suspicious nodule was analyzed. (Figure 5) 


\section{Sample collection}

Seventy-two patients who underwent thyroid surgery at Rui Jin Hospital from Sept. $2014 \sim$ Mar. 2015 because of papillary thyroid carcinoma were enrolled in this study. All these patients underwent preoperative ultrasound examination and ultrasound-guided fine needle aspiration and were pathologically diagnosed with papillary thyroid carcinoma with or without cervical lymph node metastasis. All of the patients underwent subtotal or total thyroidectomy with regional dissection, and none received chemotherapy or radiotherapy before surgery. Specimens and patient characteristics were collected. Tumor tissues were immediately frozen in liquid nitrogen and stored at $-80^{\circ} \mathrm{C}$. This study was approved by the Research Ethics Committee of our hospital, and written informed consent was obtained from all patients.

\section{Cell culture}

The human PTC cell lines TPC1 and K1 were kind gifts from the Key Laboratory for Endocrine and Metabolic Diseases of the Chinese Health Ministry (Shanghai, China). K1 is a well-differentiated PTC cell line with metastatic properties while TPC1 is a PTC cell line that does not exhibit metastatic properties [36]. TPC1 cells were cultured in RPMI 1640 medium supplemented with HEPES (Gibco, Carlsbad, CA, USA), $10 \%$ of FBS (Gibco), $100 \mathrm{U} / \mathrm{mL}$ penicillin, and $100 \mu \mathrm{g} /$ $\mathrm{mL}$ streptomycin. K1 cells were cultured in DMEM (Gibco), MCDB (Sigma, Saint Louis, Missouri, USA), and F12 (Gibco) (2:1:1) medium supplemented with $10 \%$ of FBS (Gibco), $100 \mathrm{U} / \mathrm{mL}$ penicillin, and $100 \mu \mathrm{g} /$ $\mathrm{mL}$ streptomycin. Cell lines were incubated at $37^{\circ} \mathrm{C}$ in a humidified atmosphere of $5 \% \mathrm{CO} 2$.

\section{Total RNA extraction and RT-qPCR}

Total RNA was extracted from the frozen tissues using TRIzol reagent (Invitrogen, Carlsbad, CA, USA) according to the manufacturer's protocol. One microgram of total RNA was reverse transcribed into first-strand cDNA according to the protocol of the Reverse Transcription Kit (Takara, Dalian, China). RT-qPCR was performed with a SYBR $^{\circledR}$ Premix Ex Taq ${ }^{\mathrm{TM}}$ II Kit (Takara, Dalian, China) according to the instructions in the VIIA7 system (Applied Biosystems, Foster City, CA, USA). GAPDH was used as an endogenous control for IncRNA in all amplification reactions. The primer sequences of NONHSAT076754 and GAPDH are as follows: NONHSAT076754, 5'-AAGTTTCTCACTCACCCACCTG-3' (forward), 5'- GAAGCATGTACAGTTCAGCATGTG-3' (reverse); GAPDH, 5'- AAGGTGAAGGTCGGAGTCAAC-3' (forward), 5'- GGGGTCATTGATGGCAACAATA-3' (reverse). Data were analyzed using the comparative $\mathrm{Ct}\left(2^{-\Delta \Delta \mathrm{Ct})}\right.$ method, and the value of NONHSAT076754 expression was normalized to the expression of the endogenous control. Each sample was analyzed in triplicate.

\section{Fluorescence in situ hybridization (FISH) and microscopic imaging}

The TPC1 and $\mathrm{K} 1$ cell lines were incubated at $37^{\circ} \mathrm{C}$ in a glass bottom cell culture dish (Nest Biotechnology, Wuxi, China) until they reached $60 \%$ confluence. A Cy3-fluorescence-conjugated NONHSAT076754 probe was used for RNA-FISH. A Fluorescence in Situ Hybridization Kit was purchased from RiboBio Company (Guangzhou, China). FISH was performed according to the manufacturer's protocol, and nuclei were labeled with Hoechst33342 (Thermo Fisher Scientific, Waltham, MA, USA). Fluorescence images were obtained by a laser scanning confocal microscope (LSCM) (Carl Zeiss, Oberkochen, Germany).

\section{Cell line transfection}

NONHSAT076754 was cloned into a pLVXIRES-Puro expression vector (BD Biosciences, Franklin Lakes, NJ, USA). TPC1 cells were infected with the viral suspension $24 \mathrm{~h}$ before the start of the assay. We obtained stably transfected clones by Puromycin (Promega, 2 $\mu \mathrm{g} / \mathrm{mL}$ ) selection. A stable transfectant of the pLVXIRES-Puro empty vector was used as a control. The expression of NONHSAT076754 was silenced by an antisense oligonucleotide (the antisense oligonucleotide sequence is 5'-TGATGTGGTGGTCTGGTCTC-3' while the sequence of the negative control is 5'-TCTGCTCACTTGCATGCCTT-3'). The oligonucleotides were transfected into the cells at a concentration of $100 \mathrm{nM}$ using Lipofectamine ${ }^{\mathrm{TM}} 2000$ (Thermo Fisher Scientific, Waltham, MA, USA) according to the manufacturer's instructions.

\section{Scratch assay}

The cells were incubated until they were $100 \%$ confluent and were then scratched with a pipet tip. The medium was replaced with fresh serum-free medium. The wound closing procedure was observed for $24 \mathrm{~h}$, and images were obtained.

\section{Transwell assay}

Transwell chambers (Costar, Corning, NY, USA) were used in the migration and invasion assays. Matrigel (BD Biosciences, Franklin Lakes, NJ, USA) was used 
to coat the top side of the insert membrane used in the invasion assay. Then, $200 \mu \mathrm{L}$ serum-free medium and $1 \times 10^{4}$ cells were added to the upper chamber, while $600 \mu \mathrm{L}$ medium with $5 \%$ FBS was added to the lower chamber. The chambers were maintained at $37^{\circ} \mathrm{C}$ and $5 \%$ $\mathrm{CO} 2$ for $24 \mathrm{~h}$. Next, the cells that had not migrated or invaded the top side of the insert membrane were removed with cotton swabs. The inserts were then fixed in methanol for 20 minutes and stained with $1 \%$ crystal violet for 30 minutes. The cells that had migrated or invaded the bottom of the membrane were calculated under a microscope and photographed. All experiments were performed in triplicate.

\section{Colony formation assay}

After treatment for $48 \mathrm{~h}, \mathrm{TPC} 1$ and $\mathrm{K} 1$ cells were seeded in 6-well plates at a density of 500 cells/well and cultured at $37^{\circ} \mathrm{C}$ in a humidified atmosphere of $5 \%$ $\mathrm{CO} 2$; the cultured medium was replaced every other day. After 7 days in culture, the medium was removed and the cells were washed twice with PBS. Finally, the cells were stained with crystal violet for 30 minutes at room temperature, washed again and photographed.

\section{CCK-8 analysis}

Forty-eight hours after transfection, TPC1 and K1 cells were seeded into 96 -well plates at a density of $1 \times 104$ cells/well. Cell viability was evaluated by a cell counting kit-8 assay (CCK-8; Dojindo Molecular Technologies, Japan) at 24 h, 48 h, $72 \mathrm{~h}$ and $96 \mathrm{~h}$. The absorbance at 450 $\mathrm{nm}$ was measured in a TECAN infinite M200 plate reader.

\section{Flow cytometry}

Forty-eight hours after transfection, TPC1 and $\mathrm{K} 1$ cells were collected for the cell cycle and apoptosis analyses. The apoptosis assay was performed with an Alexa Fluor $^{\circledR} 488$ Annexin V/Dead Cell Apoptosis Kit (Thermo Fisher Scientific, Waltham, MA, USA). The cells were suspended in $100 \mu \mathrm{L}$ binding buffer with $5 \mu \mathrm{L}$ Annexin $\mathrm{v}$ and $1 \mu \mathrm{L}$ propidium iodide and were incubated for 15 minutes in the dark. Then, $400 \mu \mathrm{L}$ binding buffer was added and the cells were resuspended. For the cell cycle analysis, the cells were fixed in $75 \%$ ethanol overnight at $4^{\circ} \mathrm{C}$. They were then stained using a Cell Cycle and Apoptosis Analysis Kit (Beyotime Biotechnology, Jiangsu, China) according to the manufacturer's instructions. The cells were analyzed by a Gallios Flow Cytometer (Beckman Coulter, USA) to quantify the percentage of apoptotic cells and the percentage of cells in different phases of the cell cycle. All experiments were performed independently and in triplicate.

\section{Statistical analysis}

All tests and calculations were performed with Statistical Program for Social Sciences 19.0 software (SPSS, Chicago, IL, USA) and GraphPad Prism 5.0 (GraphPad Software, LaJolla, CA, USA). Descriptive variables are presented as the mean \pm SD. Categorical variables are described as proportions. Pearson's chisquare $\left(\mathrm{X}^{2}\right)$ test was applied to examine the correlation of the NONHSAT076754 expression level with clinicopathological and ultrasonographic characteristics. An independent two-sample t test was used to compare the NONHSAT076754 expression level in the two independent groups. Logistic regression was used for the univariate analysis. The significant variables in the univariate analysis were included in the multivariate analysis. Sensitivity, specificity, positive predictive value (PPV), negative predictive value (NPV) and the diagnostic accuracy of NONHSAT076754 were assessed by ROC curve and the AUC. $P<0.05$ was considered statistically significant.

\section{ACKNOWLEDGMENTS}

The services in support of this study were provided by the Key Laboratory of Stem Cell Biology, Institute of Health Sciences, Shanghai Institutes for Biological Sciences (SIBS), Chinese Academy of Sciences (CAS). We thank Dr. Jianqiao Zhou at Ruijin Hospital for his technical assistance in image acquisition in this project.

\section{CONFLICTS OF INTERESTS}

The authors declare no competing financial interests.

\section{GRANT SUPORT}

The work was supported by the National Natural Science Foundation of China (81471668; 81671688).

\section{REFERENCES}

1. Davies L and Welch HG. Current thyroid cancer trends in the United States. JAMA otolaryngology - head \& neck surgery. 2014; 140:317-322.

2. Siegel R, Ma J, Zou Z and Jemal A. Cancer statistics, 2014. Cancer J Clin. 2014; 64:9-29.

3. O'Grady TJ, Gates MA and Boscoe FP. Thyroid cancer incidence attributable to overdiagnosis in the United States 1981-2011. International journal of cancer. 2015; 137:26642673.

4. Colonna M, Uhry Z, Guizard AV, Delafosse P, Schvartz C, Belot A, Grosclaude P and network F. Recent trends in incidence, geographical distribution, and survival of papillary thyroid cancer in France. Cancer epidemiology. 
2015; 39:511-518.

5. DeGroot LJ, Kaplan EL, McCormick M and Straus FH. Natural history, treatment, and course of papillary thyroid carcinoma. The Journal of clinical endocrinology and metabolism. 1990; 71:414-424.

6. Burman KD. Treatment of recurrent or persistent cervical node metastases in differentiated thyroid cancer: deceptively simple options. The Journal of clinical endocrinology and metabolism. 2012; 97:2623-2625.

7. Suman P, Wang CH, Abadin SS, Moo-Young TA, Prinz RA and Winchester DJ. Risk factors for central lymph node metastasis in papillary thyroid carcinoma: A National Cancer Data Base (NCDB) study. Surgery. 2016; 159:3139.

8. Xing M. Molecular pathogenesis and mechanisms of thyroid cancer. Nature reviews Cancer. 2013; 13:184-199.

9. Wang W, Larson SM, Tuttle RM, Kalaigian H, Kolbert $\mathrm{K}$, Sonenberg M and Robbins RJ. Resistance of [18f]fluorodeoxyglucose-avid metastatic thyroid cancer lesions to treatment with high-dose radioactive iodine. Thyroid. 2001; 11:1169-1175.

10. Patron V, Hitier M, Bedfert C, Le Clech G and Jegoux F. Occult lymph node metastases increase locoregional recurrence in differentiated thyroid carcinoma. The Annals of otology, rhinology, and laryngology. 2012; 121:283-290.

11. Ito $\mathrm{Y}$, Tomoda $\mathrm{C}$, Uruno $\mathrm{T}$, Takamura $\mathrm{Y}$, Miya A, Kobayashi K, Matsuzuka F, Kuma K and Miyauchi A. Clinical significance of metastasis to the central compartment from papillary microcarcinoma of the thyroid. World journal of surgery. 2006; 30:91-99.

12. Ito $\mathrm{Y}$, Tomoda $\mathrm{C}$, Uruno $\mathrm{T}$, Takamura $\mathrm{Y}$, Miya A, Kobayashi K, Matsuzuka F, Kuma K and Miyauchi A. Ultrasonographically and anatomopathologically detectable node metastases in the lateral compartment as indicators of worse relapse-free survival in patients with papillary thyroid carcinoma. World journal of surgery. 2005; 29:917-920.

13. Struhl K. Transcriptional noise and the fidelity of initiation by RNA polymerase II. Nature structural \& molecular biology. 2007; 14:103-105.

14. Gupta RA, Shah N, Wang KC, Kim J, Horlings HM, Wong DJ, Tsai MC, Hung T, Argani P, Rinn JL, Wang Y, Brzoska P, Kong B, Li R, West RB, van de Vijver MJ, et al. Long non-coding RNA HOTAIR reprograms chromatin state to promote cancer metastasis. Nature. 2010; 464:1071-1076.

15. Sun J, Song Y, Chen X, Zhao J, Gao P, Huang X, Xu H and Wang Z. Novel long non-coding RNA RP11-119F7.4 as a potential biomarker for the development and progression of gastric cancer. Oncology letters. 2015; 10:115-120.

16. Shappell SB. Clinical utility of prostate carcinoma molecular diagnostic tests. Reviews in urology. 2008; 10:44-69.

17. Panzitt K, Tschernatsch MM, Guelly C, Moustafa T, Stradner M, Strohmaier HM, Buck CR, Denk H, Schroeder R, Trauner M and Zatloukal K. Characterization of HULC, a novel gene with striking up-regulation in hepatocellular carcinoma, as noncoding RNA. Gastroenterology. 2007; 132:330-342.

18. Orozco AF and Lewis DE. Flow cytometric analysis of circulating microparticles in plasma. Cytometry Part A. 2010; 77:502-514.

19. Li-Korotky HS, Hebda PA, Lo CY and Dohar JE. Age-dependent differential expression of fibronectin variants in skin and airway mucosal wounds. Archives of otolaryngology—head \& neck surgery. 2007; 133:919-924.

20. Wang Y, Reheman A, Spring CM, Kalantari J, Marshall AH, Wolberg AS, Gross PL, Weitz JI, Rand ML, Mosher DF, Freedman J and Ni H. Plasma fibronectin supports hemostasis and regulates thrombosis. The Journal of clinical investigation. 2014; 124:4281-4293.

21. Zhang C, Wu M, Zhang L, Shang LR, Fang JH and Zhuang SM. Fibrotic microenvironment promotes the metastatic seeding of tumor cells via activating the fibronectin 1/ secreted phosphoprotein 1-integrin signaling. Oncotarget. 2016; 7:45702-45714. doi: 10.18632/oncotarget.10157.

22. Kim E, Park JS, Son KR, Kim JH, Jeon SJ and Na DG. Preoperative diagnosis of cervical metastatic lymph nodes in papillary thyroid carcinoma: comparison of ultrasound, computed tomography, and combined ultrasound with computed tomography. Thyroid. 2008; 18:411-418.

23. Chen XZ, Zhang WK, Yang K, Wang LL, Liu J, Wang L, Hu JK, Zhang B, Chen ZX, Chen JP, Zhou ZG and Mo $\mathrm{XM}$. Correlation between serum CA724 and gastric cancer: multiple analyses based on Chinese population. Molecular biology reports. 2012; 39:9031-9039.

24. Sun $Z$ and Zhang N. Clinical evaluation of CEA, CA199, CA72-4 and CA125 in gastric cancer patients with neoadjuvant chemotherapy. World journal of surgical oncology. 2014; 12:397.

25. Lee SE, Hwang TS, Choi YL, Han HS, Kim WS, Jang MH, Kim SK and Yang JH. Prognostic Significance of TERT Promoter Mutations in Papillary Thyroid Carcinomas in a BRAF(V600E) Mutation-Prevalent Population. Thyroid. 2016; 26:901-910.

26. Zhou X, Yin C, Dang Y, Ye F and Zhang G. Identification of the long non-coding RNA H19 in plasma as a novel biomarker for diagnosis of gastric cancer. Scientific reports. 2015; 5:11516.

27. Matouk IJ, Halle D, Raveh E, Gilon M, Sorin V and Hochberg A. The role of the oncofetal H19 lncRNA in tumor metastasis: orchestrating the EMT-MET decision. Oncotarget. 2016; 7:3748-3765. doi: 10.18632/ oncotarget.6387.

28. Xu S, Wang P, You Z, Meng H, Mu G, Bai X, Zhang G, Zhang $J$ and Pang D. The long non-coding RNA EPB41L4A-AS2 inhibits tumor proliferation and is associated with favorable prognoses in breast cancer and other solid tumors. Oncotarget. 2016; 7:20704-20717. doi: 10.18632 /oncotarget.8007. 
29. Faouzi M, Hague F, Geerts D, Ay AS, Potier-Cartereau M, Ahidouch A and Ouadid-Ahidouch H. Functional cooperation between KCa3.1 and TRPC1 channels in human breast cancer: Role in cell proliferation and patient prognosis. Oncotarget. 2016; 7:36419-36435. doi: 10.18632/oncotarget.9261.

30. Fradet Y, Saad F, Aprikian A, Dessureault J, Elhilali M, Trudel C, Masse B, Piche L and Chypre C. uPM3, a new molecular urine test for the detection of prostate cancer. Urology. 2004; 64:311-315; discussion 315-316.

31. Cocucci E, Racchetti G and Meldolesi J. Shedding microvesicles: artefacts no more. Trends in cell biology. 2009; 19:43-51.

32. Aribas BK, Arda K, Ciledag N, Cetindag MF, Dogan K, Sahin G, Yologlu Z and Aktas E. Fine-needle aspiration biopsy of cervical lymph nodes: factors in predicting malignant diagnosis. Neoplasma. 2011; 58:51-57.

33. Jiang B, Sun R, Fang S, Qin C, Pan X, Peng L, Li Y and
Li G. Lnc-CC3 increases metastasis in cervical cancer by increasing Slug expression. Oncotarget. 2016; 7:4165041661. doi: 10.18632/oncotarget.9519.

34. Sun TT, He J, Liang Q, Ren LL, Yan TT, Yu TC, Tang JY, Bao YJ, Hu Y, Lin Y, Sun D, Chen YX, Hong J, Chen H, Zou W and Fang JY. LncRNA GClnc1 Promotes Gastric Carcinogenesis and May Act as a Modular Scaffold of WDR5 and KAT2A Complexes to Specify the Histone Modification Pattern. Cancer discovery. 2016; 6:784-801.

35. Li W, Sun M, Zang C, Ma P, He J, Zhang M, Huang Z, Ding $Y$ and Shu Y. Upregulated long non-coding RNA AGAP2-AS1 represses LATS2 and KLF2 expression through interacting with EZH2 and LSD1 in non-small-cell lung cancer cells. Cell death \& disease. 2016; 7:e2225.

36. Saiselet M, Floor S, Tarabichi M, Dom G, Hebrant A, van Staveren WC and Maenhaut C. Thyroid cancer cell lines: an overview. Frontiers in endocrinology. 2012; 3:133. 